\title{
On the Location of the Discrete Eigenvalues for Defocusing Zakharov-Shabat Systems having Potentials with Nonvanishing Boundary Conditions
}

\author{
F. Demontis, C. van der Mee, and F. Vitale \\ ABSTRACT. In this article we prove that the discrete eigenvalues of the \\ Zakharov-Shabat system belong to certain neighborhoods of the endpoints \\ of the spectral gap and the discrete eigenvalue of the free Hamiltonian.
}

\section{Introduction}

The nonlinear Schrödinger (NLS) equation is a well-known physically and mathematically significant nonlinear evolution equation extensively studied for over forty years. For example, the NLS equation has been derived in the modeling of ocean water waves [2, 21, Bose-Einstein condensation [18, and optical fibers [10,11.

In this work we consider the defocusing NLS equation, i.e.,

$$
i q_{t}+q_{x x}-2|q|^{2} q=0
$$

[subscripts $x$ and $t$ denote partial differentiation throughout] with nonzero boundary conditions (NZBCs)

$$
q(x, t) \rightarrow q_{ \pm}(t)=q_{0} e^{2 i q_{0}^{2} t+i \theta_{ \pm}}, \text {as } x \rightarrow \pm \infty,
$$

where $i$ denotes the complex unit, $q_{0}>0$ and $0 \leq \theta_{ \pm}<2 \pi$ are arbitrary constants. It is well-known that Eq. (1.1) is associated to the so-called Zakharov-Shabat (ZS) system:

$$
\frac{\partial X}{\partial x}(x, k)=\left(-i k \sigma_{3}+Q(x)\right) X(x, k), \quad x \in \mathbb{R},
$$

where

$$
\sigma_{3}=\left(\begin{array}{cc}
1 & 0 \\
0 & -1
\end{array}\right), \quad Q(x)=\left(\begin{array}{cc}
0 & q(x) \\
q^{*}(x) & 0
\end{array}\right),
$$

$q(x)$ is the potential, $k$ is a complex spectral parameter and the asterisk denotes the complex conjugate, by means of the inverse scattering transform.

Recently, the defocusing NLS (1.1) with NZBCs has been the subject of renewed interest because of its applications to Bose-Einstein condensates $[\mathbf{8}, \mathbf{9}$ and dispersive shock waves in optical fibers [19. This justifies our effort to investigate some questions connected with this subject. In particular, we focus our attention on some aspects which arise when the Inverse Scattering Transform (IST) (see [1, 2, 20, for

2000 Mathematics Subject Classification. Primary 34A55; Secondary 78A46. 
a general review of this method) is applied to the equation (1.1) with NZBCs (1.2). In fact, the IST for (1.1) with NZBCs (see [3, 4, 7, 12, 13, 17, 22 $)$ is much more complicated than for (1.1) with decaying potentials, in particular with regard to the analyticity properties of the eigenfunctions of the scattering problem (1.3) and the corresponding scattering data. A step forward in that direction was recently made in 6 where it was proved that the direct scattering problem is well defined for potentials $q$ such that $q-q_{ \pm}$belongs to the functional class $L^{1,2}\left(\mathbb{R}^{ \pm}\right)\left(L^{1, p}\left(\mathbb{R}^{ \pm}\right)\right.$ consists of all functions $f(x)$ satisfying $\left.\int_{\mathbb{R}} d x(1+|x|)^{p}|f(x)|<\infty\right)$. For this reason, we will assume that $q-q_{ \pm} \in L^{1,2}\left(\mathbb{R}^{ \pm}\right)$.

It is also worthwhile to study if an area theorem for the defocusing NLS (1.1) with NZBCs can be proved, which means establishing existence and location of discrete eigenvalues (see Section 2 for the definition of a discrete eigenvalue) of the scattering problem (1.3) as a function of the area of the initial profile of the solution of (1.1). It is well known that for the focusing NLS with vanishing boundary conditions such a result already exists 2, 14 16]: in fact, there are no discrete eigenvalues of (1.3) if the $L^{1}$-norm of the potential is smaller than $\pi / 2$. Only recently [5], the non existence of an analogous result for equation (1.1) with NZBCs has been proved. In [5], the authors showed that no area theorem is possible for the defocusing NLS with NZBCs by providing explicit examples of box-type initial conditions where at least one discrete eigenvalue exists. In the present paper, we analyze a class of potentials more general than that considered in [5] (we only require that $q-q_{ \pm} \in L^{1,2}\left(\mathbb{R}^{ \pm}\right)$) and establish the conditions (equations (4.8) and (4.13) in Section (4) which the potentials have to satisfy in order that a particular, but well specified subset of $\left(-q_{0}, q_{0}\right)$ does not contain any discrete eigenvalue.

The paper is organized as follows. In Section 2 we review the basic facts on the direct scattering problem for (1.1) with NZBCs (1.2) and discuss an explicit example (different from that considered in 5]) which establishes the presence of at least one discrete eigenvalue in $\left(-q_{0}, q_{0}\right)$. In Section 3 we explicitly construct the resolvent of the free hamiltonian of the spectral problem originating from (1.3) and, finally, in Section 4, adapting the technique used in the vanishing case in [14 16], we state our main results, namely Theorems 4.3 and 4.4

\section{Preliminaries}

In this section we study the direct scattering problem for (1.3) by using the same notations adopted in [6] to which we refer the interested reader for details. Moreover, we discuss a significant example which shows that in the spectral gap $\left(-q_{0}, q_{0}\right)$ there may exist a discrete eigenvalue if $q_{+} \neq q_{-}$.

To study the direct scattering problem of (1.1) with NZBCs (1.2), a new spectral parameter

$$
\lambda=\sqrt{k^{2}-q_{0}^{2}}
$$

is introduced which is a conformal mapping from the Riemann $k$-surface $\mathbb{K}$ onto the Riemann $\lambda$-surface $\Lambda$. Here $\mathbb{K}$ consists of two sheets, $\mathbb{K}^{+}$and $\mathbb{K}^{-}$, which both coincide with the complex $k$-plane cut along the semilines $\Sigma=\left(-\infty,-q_{0}\right] \cup\left[q_{0},+\infty\right)$, where its edges are glued together in such a way that $\lambda(k)$ is continuous throughout the cut. The Riemann surface $\Lambda$ is the complex $\lambda$-plane consisting of the upper half-complex plane $\Lambda^{+}$and the lower half complex plane $\Lambda^{-}$glued together along 
the real $\lambda$-line. The transformation $k \mapsto \lambda$ maps $\mathbb{K}^{ \pm}$onto $\Lambda^{ \pm}$, the cut $\Sigma$ onto the real $\lambda$-line, and the points $\pm q_{0}$ to zero. Also, $\{\lambda+k, \lambda-k\} \subset \Lambda^{ \pm}$for any $k \in \mathbb{K}^{ \pm}$.

For later convenience, we write (1.3) in the form

$$
\frac{\partial X}{\partial x}(x, k)=A_{ \pm}(k) X(x, k)+\left(Q(x)-Q_{ \pm}\right) X(x, k),
$$

where

$$
A_{ \pm}(k)=-i k \sigma_{3}+Q_{ \pm}=\left(\begin{array}{cc}
-i k & q_{ \pm} \\
q_{ \pm}^{*} & i k
\end{array}\right), \quad Q_{ \pm}=\left(\begin{array}{cc}
0 & q_{ \pm} \\
q_{ \pm}^{*} & 0
\end{array}\right) .
$$

Then (1.3) and (2.1) can also be written in the equivalent form

$$
\frac{\partial X}{\partial x}(x, k)=A(x, k) X(x, k)+\left(Q(x)-Q_{f}(x)\right) X(x, k),
$$

where,

$$
A(x, k)=\theta(x) A_{+}(k)+\theta(-x) A_{-}(k), \quad Q_{f}(x)=\theta(x) Q_{+}+\theta(-x) Q_{-},
$$

and $\theta(x)$ denotes the Heaviside function defined as $\theta(x)=1$ for $x \geq 0$ and $\theta(x)=0$ for $x<0$. We associate to the ZS system (2.3) the Hamiltonian operator

$$
H=i \sigma_{3}(d / d x-Q)
$$

which is selfadjoint on the orthogonal direct sum of two copies of $L^{2}(\mathbb{R})$. It is also convenient to introduce the free Hamiltonian

$$
H_{f}=i \sigma_{3}\left(\frac{d}{d x}-Q_{f}\right) .
$$

For $k \in \Sigma$, we define the fundamental eigensolutions $\tilde{\Psi}(x, k)$ and $\tilde{\Phi}(x, k)$ as those square matrix solutions to (1.3) satisfying

$$
\begin{array}{ll}
\tilde{\Psi}(x, k)=e^{x A_{+}(k)}\left[I_{2}+o(1)\right], & x \rightarrow+\infty, \\
\tilde{\Phi}(x, k)=e^{x A_{-}(k)}\left[I_{2}+o(1)\right], & x \rightarrow-\infty .
\end{array}
$$

We can prove their existence, for $k \in \Sigma$, as the unique solutions of the Volterra integral equations [6, Prop. 1]

$$
\begin{aligned}
& \tilde{\Psi}(x, k)=\mathcal{G}(x, 0 ; k)-\int_{x}^{\infty} d y \mathcal{G}(x, y ; k)\left[Q(y)-Q_{f}(y)\right] \tilde{\Psi}(y, k), \\
& \tilde{\Phi}(x, k)=\mathcal{G}(x, 0 ; k)+\int_{-\infty}^{x} d y \mathcal{G}(x, y ; k)\left[Q(y)-Q_{f}(y)\right] \tilde{\Phi}(y, k) .
\end{aligned}
$$

Here the fundamental matrix $\mathcal{G}(x, y ; k)$ is given by

$$
\mathcal{G}(x, y ; k)= \begin{cases}e^{x A_{+}(k)} e^{-y A_{+}(k)}, & x, y \geq 0 \\ e^{x A_{+}(k)} e^{-y A_{-}(k)}, & x \geq 0 \geq y \\ e^{x A_{-}(k)} e^{-y A_{+}(k)}, & y \geq 0 \geq x \\ e^{x A_{-}(k)} e^{-y A_{-}(k)}, & x, y \leq 0\end{cases}
$$

In fact, Eqs. (2.8) are uniquely solvable under the condition $q-q_{ \pm} \in L^{1}\left(\mathbb{R}^{ \pm}\right)$if $\pm q_{0} \neq k \in \Sigma$; they are uniquely solvable under the condition $\left(1+|x|^{2}\right)\left[q-q_{ \pm}\right] \in$ $L^{1}\left(\mathbb{R}^{ \pm}\right)$if $k= \pm q_{0}$. 
Let us now introduce the Jost solutions (as column vector solutions to (2.1) in terms of the fundamental eigensolutions) and the "transition scattering" matrix $S(k)$. Hence, we define the eigenvector matrices:

$$
W_{ \pm}(k)=\left(\begin{array}{cc}
1 & \frac{-i q_{ \pm}}{\lambda+k} \\
\frac{i q_{ \pm}^{*}}{\lambda+k} & 1
\end{array}\right),
$$

where $\operatorname{det} W_{ \pm}(k)=2 \lambda /(\lambda+k)$ and $A_{ \pm}(k) W_{ \pm}(k)=W_{ \pm}(k) \operatorname{diag}(-i \lambda, i \lambda)$. The Jost solutions from the right and the left, respectively, are defined as the columns of

$$
\tilde{\Psi}(x, k) W_{+}(k)=(\bar{\psi}(x, k) \quad \psi(x, k)), \quad \tilde{\Phi}(x, k) W_{-}(k)=(\phi(x, k) \quad \bar{\phi}(x, k)),
$$

and a detailed study of their analyticity properties can be found in [6. Prop. 3]. Since $\tilde{\Psi}(x, k)$ and $\tilde{\Phi}(x, k)$ are square matrix solutions of the homogeneous first order system (1.3), we have

$$
\tilde{\Psi}(x, k)=\tilde{\Phi}(x, k) \mathbb{A}_{l}(k), \quad \tilde{\Phi}(x, k)=\tilde{\Psi}(x, k) \mathbb{A}_{r}(k),
$$

where $\mathbb{A}_{l}(k)$ and $\mathbb{A}_{r}(k)$ are called the transition coefficient matrices whose expressions are given by

$$
\begin{aligned}
& \mathbb{A}_{l}(k)=I_{2}-\int_{-\infty}^{\infty} d y \mathcal{G}(0, y ; k)\left[Q(y)-Q_{f}(y)\right] \tilde{\Psi}(y, k), \\
& \mathbb{A}_{r}(k)=I_{2}+\int_{-\infty}^{\infty} d y \mathcal{G}(0, y ; k)\left[Q(y)-Q_{f}(y)\right] \tilde{\Phi}(y, k) .
\end{aligned}
$$

As a result of (2.11) and (2.12), we get

$$
\begin{aligned}
& (\phi(x, k) \quad \bar{\phi}(x, k))=(\bar{\psi}(x, k) \quad \psi(x, k)) S(k), \\
& (\bar{\psi}(x, k) \quad \psi(x, k))=(\phi(x, k) \quad \bar{\phi}(x, k)) \bar{S}(k),
\end{aligned}
$$

where 6 .

$$
S(k)=W_{+}^{-1}(k) \mathbb{A}_{r}(k) W_{-}(k)=\left(\begin{array}{cc}
a(k) & \bar{b}(k) \\
b(k) & \bar{a}(k)
\end{array}\right),
$$

and $\bar{S}(k)=W_{-}^{-1}(k) \mathbb{A}_{l}(k) W_{+}(k)=S^{-1}(k)$. The analyticity and continuity properties of the scattering coefficients $a(k), \bar{b}(k), b(k), \bar{a}(k)$ follow from the analyticity and continuity properties of the Jost solutions using their Wronskian representations [6]. It is well known that the scattering data associated to the ZS system (1.3) are (see $[\mathbf{1}, \mathbf{2}, \mathbf{2 0}, \mathbf{2 2})$ ): the reflection coefficient $\rho(k)=b(k) / a(k)$, the zeros of $a(k)$ for $k \in \mathbb{K} \backslash \Sigma$ (the so-called discrete eigenvalues) and a suitable set of constants associated with the discrete eigenvalues known as norming constants. However, it is important to remark that for Eq. (1.1) with NZBCs, the discrete eigenvalues belong to the spectral gap $\left(-q_{0}, q_{0}\right)$ and are simple (proved in [7]) and, under the hypothesis $q-q_{ \pm} \in L^{1,4}\left(\mathbb{R}^{ \pm}\right)$, are finite in number (established in [6]).

We conclude this section analyzing an explicit example which confirms the results obtained in [5, Sec. 4]

ExAmple 2.1 (Free Hamiltonian). Let us compute the discrete eigenvalues of the free Hamiltonian introduced in (2.6) [which corresponds to assume $Q(x)=$ $Q_{f}(x)$ in the ZS system (1.3)]. In that case from (2.13) we have $\mathbb{A}_{l}(k)=\mathbb{A}_{r}(k)=I_{2}$ 
[ $I_{2}$ denotes the $2 \times 2$ identity matrix], and by using (2.12) we get

$$
\tilde{\Psi}(x, k)=\tilde{\Phi}(x, k)= \begin{cases}e^{x A_{+}(k)}, & \text { for } x>0, \\ e^{x A_{-}(k)}, & \text { for } x<0 .\end{cases}
$$

From (2.16) and taking into account (2.15) and (2.10), we arrive at

$$
S(k)=\left(\begin{array}{cc}
a(k) & \bar{b}(k) \\
b(k) & \bar{a}(k)
\end{array}\right)=W_{+}^{-1}(k) W_{-}(k)=\frac{\lambda+k}{2 \lambda}\left(\begin{array}{cc}
1-\frac{q_{+} q_{-}^{*}}{(\lambda+k)^{2}} & i \frac{\left(q_{+}-q_{-}\right)}{\lambda+k} \\
i \frac{\left(q_{-}^{*}-q_{+}^{*}\right)}{\lambda+k} & 1-\frac{q_{-} q_{+}^{*}}{(\lambda+k)^{2}}
\end{array}\right) .
$$

Putting $\lambda=i \ell$ with $\ell \in\left(0, q_{0}\right)$, from the preceding equation we obtain:

$$
a(k)=\frac{(k+i \ell)^{2}-q_{0}^{2} e^{i\left(\theta_{+}-\theta_{-}\right)}}{2 i \ell(k+i \ell)}=\frac{k^{2}-\ell^{2}-q_{0}^{2} \cos \left(\theta_{+}-\theta_{-}\right)+i\left(2 k \ell-q_{0}^{2} \sin \left(\theta_{+}-\theta_{-}\right)\right)}{2 i \ell(k+i \ell)} .
$$

As a result $a(k)=0$ if and only if

$$
\cos \left(\theta_{+}-\theta_{-}\right)=\frac{k^{2}-\ell^{2}}{q_{0}^{2}}, \quad \sin \left(\theta_{+}-\theta_{-}\right)=\frac{2 k \ell}{q_{0}^{2}} .
$$

Equation (2.17) has a unique solution $k_{0} \in\left(-q_{0}, q_{0}\right)$, unless $q_{+}=q_{-}$. This eigenvalue $k_{0}=0$ iff $\theta_{+}-\theta_{-}$is an odd multiple of $\pi$.

From now on, we denote with $k_{0}$ the unique discrete eigenvalue of the free Hamiltonian operator associated to the scattering problem (1.3) and computed in the example above.

\section{Resolvent of the free Hamiltonian}

In this section we calculate the resolvent $\left(k-H_{f}\right)^{-1}$ of the free Hamiltonian $H_{f}$. The result obtained will be used in the next section to determine the location of the discrete eigenvalues.

Let us compute the resolvent $\left(k-H_{f}\right)^{-1}$ of the free Hamiltonian for $k \notin$ $\sigma\left(H_{f}\right)=\Sigma \cup\left\{k_{0}\right\}$ if $q_{+} \neq q_{-}$[or $k \notin \sigma\left(H_{f}\right)=\Sigma$ if $\left.q_{+}=q_{-}\right]$. Letting $F(x)$ be a column vector function in $L^{2}(\mathbb{R})^{2}=L^{2}(\mathbb{R}) \oplus L^{2}(\mathbb{R})$, we seek the unique vector function $\Psi(k, x)$ in $L^{2}(\mathbb{R})^{2}$ such that $H_{f} \Psi=k \Psi-F$. Writing $\Psi(x, k)=$ $W(x, k) \psi(x, k)$, where $W(x, k)=W_{+}(k) \theta(x)+W_{-}(k) \theta(-x)$, we get for $0 \neq x \in \mathbb{R}$

$$
\psi^{\prime}(x, k)=-i \lambda \sigma_{3} \psi(x, k)+i W^{-1}(x, k) \sigma_{3} F(x),
$$

where we have used that $A(x, k) W(x, k)=-i \lambda W(x, k) \sigma_{3}$.

TheOREM 3.1. Let $q_{+} \neq q_{-}$and $k \in \mathbb{K}^{+}$. Then for $F \in L^{2}(\mathbb{R})^{2}$ and $k \notin$ $\Sigma \cup\left\{k_{0}\right\}$ we have

$$
\left[\left(k-H_{f}\right)^{-1} F\right](x)=\int_{-\infty}^{\infty} d y\left[K_{f}(x, y ; k)+K_{f 1}(x ; k) K_{f 2}(y ; k)\right] F(y),
$$


where $K_{f}(x, y ; k)$ is given by

$$
\begin{aligned}
& K_{f}(x, y ; k)= \begin{cases}-i e^{i \lambda(y-x)} W_{+}(k)\left(\begin{array}{ll}
1 & 0 \\
0 & 0
\end{array}\right) \sigma_{3} W_{+}^{-1}(k) \sigma_{3}, & y>x>0, \\
-i e^{i \lambda(x-y)} W_{+}(k)\left(\begin{array}{ll}
0 & 0 \\
0 & 1
\end{array}\right) \sigma_{3} W_{+}^{-1}(k) \sigma_{3}, & x>y>0, \\
-i e^{i \lambda(y-x)} W_{-}(k)\left(\begin{array}{ll}
1 & 0 \\
0 & 0
\end{array}\right) \sigma_{3} W_{-}^{-1}(k) \sigma_{3}, & 0>y>x, \\
-i e^{i \lambda(x-y)} W_{-}(k)\left(\begin{array}{ll}
0 & 0 \\
0 & 1
\end{array}\right) \sigma_{3} W_{-}^{-1}(k) \sigma_{3}, & 0>x>y, \\
0, & x y<0,\end{cases} \\
& K_{f 1}(x ; k)= \begin{cases}e^{i \lambda x}\left(\begin{array}{ll}
0 & 0 \\
0 & 1
\end{array}\right) W_{+}^{-1}(k), & x>0, \\
e^{-i \lambda x}\left(\begin{array}{ll}
1 & 0 \\
0 & 0
\end{array}\right) W_{-}^{-1}(k), & x<0\end{cases} \\
& K_{f 2}(y ; k)= \begin{cases}-i e^{i \lambda y} Z^{-1}(k)\left(\begin{array}{cc}
1 & 0 \\
0 & 0
\end{array}\right) W_{+}^{-1}(k) \sigma_{3}, & y>0, \\
i e^{-i \lambda y} Z^{-1}(k)\left(\begin{array}{cc}
0 & 0 \\
0 & 1
\end{array}\right) W_{-}^{-1}(k) \sigma_{3}, & y<0,\end{cases}
\end{aligned}
$$

$$
Z(k)=\left(\begin{array}{cc}
1 & 0 \\
0 & 0
\end{array}\right) W_{+}^{-1}(k)+\left(\begin{array}{cc}
0 & 0 \\
0 & 1
\end{array}\right) W_{-}^{-1}(k)=\frac{1}{2 \lambda}\left(\begin{array}{cc}
\lambda+k & i q_{r} \\
-i q_{l}^{*} & \lambda+k
\end{array}\right) .
$$

If $q_{+}=q_{-} \neq 0$, then for $k \notin \Sigma$

$$
\left[\left(k-H_{f}\right)^{-1} F\right](x)=-i \int_{-\infty}^{\infty} d y e^{i \lambda|x-y|} W_{+}(k) E(y-x) W_{+}^{-1}(k) \sigma_{3} F(y),
$$

where

$$
E(w)=\theta(w)\left(\begin{array}{ll}
1 & 0 \\
0 & 0
\end{array}\right)+\theta(-w)\left(\begin{array}{cc}
0 & 0 \\
0 & -1
\end{array}\right)
$$

A similar result can be proved also if $k \in \mathbb{K}^{-}$(or $\lambda \in \Lambda^{-}$).

Proof. Let us assume that $q_{+} \neq q_{-}$. Then (3.1) implies the identity

$$
\frac{\partial}{\partial y}\left\{e^{-i \lambda(x-y) \sigma_{3}} \psi(y, k)\right\}=i e^{-i \lambda(x-y) \sigma_{3}} W^{-1}(y, k) \sigma_{3} F(y),
$$

where $0 \neq y \in \mathbb{R}$ and $k \notin \Sigma$. Therefore, for $0 \neq y \in \mathbb{R}$ and $k \notin \Sigma$ we obtain

$$
\begin{aligned}
& \frac{\partial}{\partial y}\left\{e^{i \lambda(y-x)}\left(\begin{array}{ll}
1 & 0 \\
0 & 0
\end{array}\right) \psi(y, k)\right\}=i e^{i \lambda(y-x)}\left(\begin{array}{ll}
1 & 0 \\
0 & 0
\end{array}\right) W^{-1}(y, k) \sigma_{3} F(y), \\
& \frac{\partial}{\partial y}\left\{e^{i \lambda(x-y)}\left(\begin{array}{ll}
0 & 0 \\
0 & 1
\end{array}\right) \psi(y, k)\right\}=i e^{i \lambda(x-y)}\left(\begin{array}{ll}
0 & 0 \\
0 & 1
\end{array}\right) W^{-1}(y, k) \sigma_{3} F(y) .
\end{aligned}
$$

Let $k$ belong to $\mathbb{K}^{+}$[or $\lambda \in \Lambda^{+}$] and let us integrate (3.5a) with respect to $y \in$ $(x,+\infty)$ for $x \geq 0$ and with respect to $y \in(x, 0)$ for $x<0$ and $(3.5 \mathrm{~b}$ ) with respect to $y \in(-\infty, x)$ for $x \leq 0$ and with respect to $y \in(0, x)$ for $x>0$. Putting together 
the results obtained in this way for $x>0$ and $x<0$, respectively, we obtain $[\Psi(x, k)$ is continuous in $x=0$ but $\psi(x, k)$ is not]

$$
\begin{aligned}
\Psi(x, k) & =-i W_{+}(k) \int_{x}^{\infty} d y e^{i \lambda(y-x)}\left(\begin{array}{cc}
1 & 0 \\
0 & 0
\end{array}\right) W_{+}^{-1}(k) \sigma_{3} F(y) \\
& +i W_{+}(k) \int_{0}^{x} d y e^{i \lambda(x-y)}\left(\begin{array}{cc}
0 & 0 \\
0 & 1
\end{array}\right) W_{+}^{-1}(k) \sigma_{3} F(y) \\
& +e^{i \lambda x} W_{+}(k)\left(\begin{array}{cc}
0 & 0 \\
0 & 1
\end{array}\right) W_{+}^{-1}(k) \Psi(0, k) \quad \text { for } x>0, \\
& =i W_{-}(k) \int_{-\infty}^{x} d y e^{i \lambda(x-y)}\left(\begin{array}{cc}
0 & 0 \\
0 & 1
\end{array}\right) W_{-}^{-1}(k) \sigma_{3} F(y) \\
& -i W_{-}(k) \int_{x}^{0} d y e^{i \lambda(y-x)}\left(\begin{array}{cc}
1 & 0 \\
0 & 0
\end{array}\right) W_{-}^{-1}(k) \sigma_{3} F(y) \\
& +e^{-i \lambda x} W_{-}(k)\left(\begin{array}{cc}
1 & 0 \\
0 & 0
\end{array}\right) W_{-}^{-1}(k) \Psi(0, k) \quad \text { for } x<0, \\
& =Z^{-1}(k)\left[-i \int_{0}^{\infty} d y e^{i \lambda y}\left(\begin{array}{cc}
1 & 0 \\
0 & 0
\end{array}\right) W_{+}^{-1}(k) \sigma_{3} F(y)\right. \\
& \left.+i \int_{-\infty}^{0} d y e^{-i \lambda y}\left(\begin{array}{cc}
0 & 0 \\
0 & 1
\end{array}\right) W_{-}^{-1}(k) \sigma_{3} F(y)\right] \quad \text { for } x=0,
\end{aligned}
$$

provided the matrix $Z(k)$ is nonsingular. Putting $k \in\left(-q_{0}, q_{0}\right)$ and $\lambda=i \ell$, we get

$$
\operatorname{det} Z(k)=\frac{\ell^{2}-k^{2}+q_{0}^{2} \cos \left(\theta_{+}-\theta_{-}\right)+i\left[-2 k \ell+q_{0}^{2} \sin \left(\theta_{+}-\theta_{-}\right)\right]}{4 \ell^{2}},
$$

which can only vanish if (2.17) is true. In other words, in the case $q_{+} \neq q_{-}$we have to exclude the discrete eigenvalue $k=k_{0}$.

Let us now consider the case $q_{+}=q_{-}$. Writing $\Psi(x, k)=W_{+}(k) \psi(x, k)$ for the left-hand side of (3.4), we verify that it satisfies

$$
\Psi^{\prime}(x, k)=A_{+}(k) \Psi(x, k)+i \sigma_{3} F(x) .
$$

and formula (3.4) follows from (3.3a) $-3.3 \mathrm{~d}$ ) if one takes into account that $q_{+}=q_{-}$ implies $W_{+}=W_{-}$and this completes the proof.

We underline that:

a. If $q_{+} \neq q_{-}$, the resolvent operator $\left(k-H_{f}\right)^{-1}$ is the sum of the integral operator with integral kernel $K_{f}(x, y ; k)$ for $k \notin \Sigma$ and a rank two operator for $k \notin \Sigma \cup\left\{k_{0}\right\}$;

b. If $q_{+}=q_{-}$the resolvent operator $\left(k-H_{f}\right)^{-1}$ is the integral operator with integral kernel $-i e^{i \lambda|x-y|} W_{+}(k) E(y-x) W_{+}^{-1}(k) \sigma_{3}$.

\section{Location of the discrete eigenvalues}

The aim of this section is to characterize the location of the discrete eigenvalues in $\left(-q_{0}, q_{0}\right)$. In fact, Example 2.1 shows that if $q_{-} \neq q_{+}$at least one such eigenvalue exists. We arrive at our main results mimicking the proofs given by Klaus et al. in 14 16 for focusing NLS with decaying potential. We need the following technical results, i.e., Theorems 4.1 and 4.2 below. 
THEOREM 4.1. Let $W^{(1)}(x)$ and $W^{(2)}(x)$ be two $2 \times 2$ matrices whose entries belong to $L^{2}(\mathbb{R})$, and let $k \notin \Sigma$. Then the integral operator on $L^{2}(\mathbb{R})^{2}$ with integral kernel

$$
W^{(1)}(x) K_{f}(x, y ; k) W^{(2)}(y)
$$

is Hilbert-Schmidt, also if we take the limit as $k$ approaches an interior point of $\Sigma$.

Proof. The squared Hilbert-Schmidt norm of $W^{(1)}\left(k-H_{f}\right)^{-1} W^{(2)}$ minus the rank two contribution is given by

$$
\int_{-\infty}^{\infty} d x \int_{-\infty}^{\infty} d y\left\|W^{(1)}(x)\right\|_{\mathrm{HS}}^{2}\left\|W^{(2)}(y)\right\|_{\mathrm{H} S}^{2}\left|K_{f}(x, y ; k)\right|^{2} .
$$

As the exponential factor $e^{i \lambda|x-y|}$ in the expression for $K_{f}(x, y ; k)$ is bounded above by 1 in absolute value, we get the following upper bound for the squared HilbertSchmidt norm of $W^{(1)}\left(k-H_{f}\right)^{-1} W^{(2)}$ minus the rank two contribution:

$$
\frac{k^{2}}{\lambda^{2}}\left(\int_{-\infty}^{\infty} d x\left\|W^{(1)}(x)\right\|_{\mathrm{HS}}^{2}\right)\left(\int_{-\infty}^{\infty} d y\left\|W^{(2)}(y)\right\|_{\mathrm{HS}}^{2}\right),
$$

which, for each $\varepsilon>0$, is uniformly bounded in $k$ satisfying $\sqrt{\left|k^{2}-q_{0}^{2}\right|} \geq \varepsilon$.

The following theorem is immediate as a consequence of the definition of the Hilbert-Schmidt norm. In the norm estimate it does not matter which unitarily equivalent matrix norm is taken, because $K_{f 1}(x ; k)$ and $K_{f 2}(y ; k)$ both have rank one.

THEOREM 4.2. Let $W^{(1)}(x)$ and $W^{(2)}(x)$ be two $2 \times 2$ matrices whose entries belong to $L^{2}(\mathbb{R})$, and let $k \notin \Sigma$. Then the integral operator on $L^{2}(\mathbb{R})^{2}$ with integral kernel

$$
W^{(1)}(x) K_{f 1}(x ; k) K_{f 2}(y ; k) W^{(2)}(y)
$$

is rank two and its norm coincides with

$$
\left[\int_{-\infty}^{\infty} d x\left\|W^{(1)}(x) K_{f 1}(x ; k)\right\|^{2}\right]^{1 / 2}\left[\int_{-\infty}^{\infty} d y\left\|K_{f 2}(y ; k) W^{(2)}(y)\right\|^{2}\right]^{1 / 2} .
$$

It follows from [14 Eq. (4.10)] and [16, proof of Thm. 4.2] that the norm of the integral operator on $L^{2}(\mathbb{R})$ with kernel $e^{-\tau(y-x)} \theta(y-x) \sqrt{m(x) m(y)}$ is less than $(2 / \pi)\|m\|_{1}$. To generalize this result to the nonvanishing case, we start with the polar decomposition

$$
\begin{aligned}
Q(x)-Q_{f}(x) & =\left(\begin{array}{cc}
0 & \sigma(x) \\
\sigma(x)^{*} & 0
\end{array}\right) \sqrt{\left|q(x)-q_{f}(x)\right|} I_{2} \cdot \sqrt{\left|q(x)-q_{f}(x)\right|} I_{2} \\
& =U_{\delta}(x)|\Delta(x)|^{1 / 2}|\Delta(x)|^{1 / 2}
\end{aligned}
$$

where

$$
q_{f}(x)=\left(q(x)-q_{-}\right) \theta(-x)+\left(q(x)-q_{+}\right) \theta(x), \quad \Delta(x)=\left|q(x)-q_{f}(x)\right| I_{2},
$$

$|\sigma(x)|=1$, and hence $U_{\delta}(x)=\left(\begin{array}{cc}0 & \sigma(x) \\ \sigma(x)^{*} & 0\end{array}\right)$ is a unitary matrix. For $k \notin \Sigma$ we now define

$$
\mathcal{W}(k)=I+|\Delta|^{1 / 2}\left(k-H_{f}\right)^{-1} U_{\delta}|\Delta|^{1 / 2}
$$


Then, for $k \notin \Sigma$ [and for $k \neq k_{0}$ if $q_{+} \neq q_{-}$], $\mathcal{W}(k)-I$ is a Hilbert-Schmidt operator on $L^{2}(\mathbb{R})^{2}$ with integral kernel

$$
\begin{cases}|\Delta(x)|^{1 / 2}\left[K_{f}(x, y ; k)+K_{f 1}(x ; k) K_{f 2}(y ; k)\right] U_{\delta}(y)|\Delta(y)|^{1 / 2}, & q_{+} \neq q_{-}, \\ -i e^{i \lambda|x-y|} W_{+}(k) E(y-x) W_{+}^{-1}(k) \sigma_{3}, & q_{+}=q_{-} .\end{cases}
$$

It is now verified that 1

$$
(k-H)^{-1}-\left(k-H_{f}\right)^{-1}=-\left(k-H_{f}\right)^{-1} U_{\delta}|\Delta|^{1 / 2} \mathcal{W}^{-1}(k)|\Delta|^{1 / 2}\left(k-H_{f}\right)^{-1}
$$

provided $\mathcal{W}(k)$ is invertible. The right-hand side of this identity has a finite limit as $k$ approaches the interior points of $\Sigma$, provided $\mathcal{W}(k)$ is invertible 2 For $k_{0} \neq k \notin \Sigma$, the points of noninvertibility of $\mathcal{W}(k)$ are exactly the discrete eigenvalues.

Our first result is as follows:

THEOREM 4.3. Let us consider the case $q_{+} \neq q_{-}$. Then, for $k \in\left(-q_{0}, q_{0}\right)$, $\mathcal{W}(k)$ is invertible if the right-hand side of equation (4.8)

$$
\|\mathcal{W}(k)-I\| \leq\left\|q-q_{f}\right\|_{1}\left|\frac{k(k+\lambda)}{2 \lambda^{2} \operatorname{det} Z(k)}\right|\left\{\frac{2}{\pi}+\sqrt{\left|\frac{k(k+\lambda)}{2 \lambda^{2}}\right|}\right\} .
$$

is strictly less than one. As a consequence, the discrete eigenvalues belong to these neighborhoods of $q_{0},-q_{0}$, and $k_{0}$ within $\left(-q_{0}, q_{0}\right)$ for which the right-hand side of (4.8) is at least one.

Proof. As already noted at the end of Section 3, when $q_{+} \neq q_{-}$the resolvent operator $\left(k-H_{f}\right)^{-1}$ is the sum of the integral operator with integral kernel $K_{f}(x, y ; k)$ for $k \notin \Sigma$ and a rank two operator for $k \notin \Sigma \cup\left\{k_{0}\right\}$. The idea is to estimate the integral kernel $K_{f}(x, y ; k)$ by using (4.1) and the rank two contribution through (4.2). To use equations (4.1) and (4.2), we introduce $W^{(1)}(x)$ and $W^{(2)}(y)$ as

$$
\begin{aligned}
& W^{(1)}(x)=|\Delta(x)|^{1 / 2}=\sqrt{\left|q(x)-q_{f}(x)\right|} I_{2}, \\
& W^{(2)}(y)=U_{\delta}(y)|\Delta(y)|^{1 / 2}=\sqrt{\left|q(y)-q_{f}(y)\right|}\left(\begin{array}{cc}
0 & \sigma(y) \\
\sigma(y)^{*} & 0
\end{array}\right) .
\end{aligned}
$$

First of all, it is easily verified that $W^{(1)}(x) K_{f 1}(x ; k)$ has as its (spectral) norm

$$
e^{-|x| \operatorname{Im} \lambda} \sqrt{\left|q(x)-q_{f}(x)\right|} \sqrt{\left|\frac{k(k+\lambda)}{2 \lambda^{2}}\right|} .
$$

By straightforward calculations, we also get the (spectral) norm of $K_{f 2}(y ; k) W^{(2)}(y)$

$$
e^{-|y| \operatorname{Im} \lambda} \sqrt{\left|q(y)-q_{f}(y)\right|}\left|\frac{k(k+\lambda)}{2 \lambda^{2} \operatorname{det} Z(k)}\right|,
$$

and, finally,

$$
\left|\frac{K_{f}(x, y ; k)}{e^{i \lambda|x-y|}}\right|=\left|\frac{k}{\lambda}\right|
$$

\footnotetext{
${ }^{1}$ By using eqs. (4.3) and (4.5) and that $k I-H=k I-H_{f}+Q-Q_{f}$, long but straightforward calculations show that the right and the left hand side of the equation obtained by multiplying both member of (4.7) by $k I-H$ coincide and then identity (4.7) holds.

${ }^{2}$ For $k$ an interior point of $\Sigma$, the points of noninvertibility of $\mathcal{W}(k)$ would be the spectral singularities, but they are known not to exist [6].
} 
Applying (4.10), (4.11) and (4.12) to (4.1), it is easily verified that the integral operator on $L^{2}(\mathbb{R})$ has the integral kernel in the form $e^{-\tau(y-x)} \theta(y-x) \sqrt{m(x) m(y)}$ which has, for $\tau \geq 0$, norm at most $(2 / \pi)\|m\|_{1}$ (as stated above). Thus, $\mathcal{W}(k)-I$ minus the rank two contribution has norm at most

$$
\frac{2}{\pi}\left\|q-q_{f}\right\|_{1}\left|\frac{k(k+\lambda)}{2 \lambda^{2} \operatorname{det} Z(k)}\right| \text {. }
$$

By using (4.2), we also verify that the rank two contribution to $\mathcal{W}(k)-I$ has norm at most

As a result,

$$
\left\|q-q_{f}\right\|_{1} \sqrt{\left|\frac{k(k+\lambda)}{2 \lambda^{2}}\right|}\left|\frac{k(k+\lambda)}{2 \lambda^{2} \operatorname{det} Z(k)}\right| .
$$

$$
\|\mathcal{W}(k)-I\| \leq\left\|q-q_{f}\right\|_{1}\left|\frac{k(k+\lambda)}{2 \lambda^{2} \operatorname{det} Z(k)}\right|\left\{\frac{2}{\pi}+\sqrt{\left|\frac{k(k+\lambda)}{2 \lambda^{2}}\right|}\right\},
$$

which completes the proof.

The next theorem sheds light on the case $q_{+}=q_{-}$.

THEOREM 4.4. Let $q_{+}=q_{-}$. Then, for $k \in\left(-q_{0}, q_{0}\right), \mathcal{W}(k)$ is invertible if the right-hand side of equation 4.13)

$$
\|\mathcal{W}(k)-I\| \leq \frac{2}{\pi}\left\|q-q_{f}\right\|_{1}\left|\frac{k}{\lambda}\right| .
$$

is strictly less than one. As a consequence, the discrete eigenvalues belong to these neighborhoods of $q_{0},-q_{0}$ for which the right-hand side of (4.13) is at least one.

Proof. Equation (4.13) immediately follows from the second of (4.6) taking into account (4.10), (4.11) and (4.12).

We remark that these results agree with those found in [5, Sec. 4].

Finally, for $q_{+} \neq q_{-}$we prove the existence of a discrete eigenvalue in the spectral gap if $\left\|q-q_{f}\right\|_{1}$ is sufficiently small.

Theorem 4.5. Let $q_{+} \neq q_{-}$and let $\left(k_{0}-\epsilon, k_{0}+\epsilon\right) \subseteq\left(-q_{0}, q_{0}\right)$. Put $C_{\epsilon}=$ $\max _{\left|k-k_{0}\right|=\epsilon}\left(\left\|\left(k-H_{f}\right)^{-1}\right\|^{2}\left\|\mathcal{W}^{-1}(k)\right\|\right)$. Then for

$$
\left\|q-q_{f}\right\|_{1}<\frac{1}{\epsilon C_{\epsilon}}
$$

there exists a simple discrete eigenvalue $k \in\left(k_{0}-\epsilon, k_{0}+\epsilon\right)$.

Proof. Let $\Gamma(\epsilon)$ be the positively oriented circle with center $k_{0}$ and radius $\epsilon$. Using (4.7) and $\left\||\Delta|^{\frac{1}{2}}\right\|^{2}=\left\|q-q_{f}\right\|_{1}$ (where $|\Delta|$ is defined by (4.4)), we estimate

$$
\left\|\frac{1}{2 \pi i} \int_{\Gamma(\epsilon)} d k\left\{(k-H)^{-1}-\left(k-H_{f}\right)^{-1}\right\}\right\| \leq \epsilon\left\|q-q_{f}\right\|_{1} C_{\epsilon}<1
$$

whenever $\left\|q-q_{f}\right\|_{1}<\frac{1}{\epsilon C_{\epsilon}}$. Now recall that $P=\frac{1}{2 \pi i} \int_{\Gamma(\epsilon)} d k(k-H)^{-1}$ and $P_{f}=$ $\frac{1}{2 \pi i} \int_{\Gamma(\epsilon)} d k\left(k-H_{f}\right)^{-1}$ are the orthogonal projections onto the combined eigenvector subspaces of $H$ and $H_{f}$ corresponding to the eigenvalues in $\left(k_{0}-\epsilon, k_{0}+\epsilon\right)$. We have proved above that

$$
\left\|P-P_{f}\right\|<1
$$


Since $I+P-P_{f}$ and $I-P+P_{f}$ are nonsingular and

$$
\left(I+P-P_{f}\right) P_{f}=P P_{f}=P\left(I-P+P_{f}\right),
$$

the projections $P$ and $P_{f}$ have the same rank, which equals +1 . This completes the proof.

\section{Acknowledgements}

The research leading to this article was supported in part by INdAM-GNFM, INFN IS-CSN4, and MIUR under the PRIN 2011 grant Teorie geometriche $e$ analitiche dei sistemi Hamiltoniani in dimensioni finite e infinite.

\section{References}

[1] M. J. Ablowitz, B. Prinari, and A. D. Trubatch, Discrete and continuous nonlinear Schrödinger systems, London Mathematical Society Lecture Note Series, vol. 302, Cambridge University Press, Cambridge, 2004. MR2040621 (2005c:37117)

[2] Mark J. Ablowitz and Harvey Segur, Solitons and the inverse scattering transform, SIAM Studies in Applied Mathematics, vol. 4, Society for Industrial and Applied Mathematics (SIAM), Philadelphia, Pa., 1981. MR642018 (84a:35251)

[3] Naruyosi Asano and Yusuke Kato, Nonselfadjoint Zakharov-Shabat operator with a potential of the finite asymptotic values. I. Direct spectral and scattering problems, J. Math. Phys. 22 (1981), no. 12, 2780-2793, DOI 10.1063/1.525185. MR638083 (83a:35086)

[4] Naruyoshi Asano and Yusuke Kato, Nonselfadjoint Zakharov-Shabat operator with a potential of the finite asymptotic values. II. Inverse problem, J. Math. Phys. 25 (1984), no. 3, 570-588, DOI 10.1063/1.526181. MR737304 (86c:35128)

[5] Gino Biondini and Barbara Prinari, On the spectrum of the Dirac operator and the existence of discrete eigenvalues for the defocusing nonlinear Schrödinger equation, Stud. Appl. Math. 132 (2014), no. 2, 138-159, DOI 10.1111/sapm.12024. MR.3167091

[6] F. Demontis, B. Prinari, C. van der Mee, and F. Vitale, The inverse scattering transform for the defocusing nonlinear Schrödinger equations with nonzero boundary conditions, Stud. Appl. Math. 131 (2013), no. 1, 1-40, DOI 10.1111/j.1467-9590.2012.00572.x. MR3081238

[7] L. D. Faddeev and L. A. Takhtajan, Hamiltonian methods in the theory of solitons, Springer Series in Soviet Mathematics, Springer-Verlag, Berlin, 1987. Translated from the Russian by A. G. Reyman [A. G. Reĭman]. MR905674 (89m:58103)

[8] C. Hamner, J.J. Chang, P. Engels, and M. Hoefer, Generation of dark-bright soliton trains in superfluid-superfluid counterflow, Phys. Rev. Lett. 106:065302 (2011).

[9] D. Yan, J.J. Chang, C. Hamner, P.G. Kevrekidis, P. Engels, V. Achilleos, D.J. Frantzeskakis, R. Carretero-Gonzáles, and P. Schmelcher, Multiple dark-bright solitons in atomic BoseEinstein condensates, Phys. Rev. A 84:053630 (2011).

[10] A. Hasegawa and F. Tappert, Transmission of stationary nonlinear optical pulses in dispersive dielectric fibers. I. Anomalous dispersion, App. Phys. Lett. 23(3) 142-144 (1973).

[11] A. Hasegawa and F. Tappert, Transmission of stationary nonlinear optical pulses in dispersive dielectric fibers. II. Normal dispersion, App. Phys. Lett. 23(3) 171-172 (1973).

[12] Tutomu Kawata and Hiroshi Inoue, Eigen value problem with nonvanishing potentials, J. Phys. Soc. Japan 43 (1977), no. 1, 361-362. MR.0450809 (56 \#9102)

[13] Tutomu Kawata and Hiroshi Inoue, Inverse scattering method for the nonlinear evolution equations under nonvanishing conditions, J. Phys. Soc. Japan 44 (1978), no. 5, 1722-1729. MR0499839 (58 \#17600)

[14] Martin Klaus, On the Zakharov-Shabat eigenvalue problem, Mathematical studies in nonlinear wave propagation, Contemp. Math., vol. 379, Amer. Math. Soc., Providence, RI, 2005, pp. 2145, DOI 10.1090/conm/379/07023. MR2149044(2006k:34232)

[15] Martin Klaus, On the eigenvalues of the Lax operator for the matrix-valued AKNS system, Topics in operator theory. Volume 2. Systems and mathematical physics, Oper. Theory Adv. Appl., vol. 203, Birkhäuser Verlag, Basel, 2010, pp. 289-323, DOI 10.1007/978-3-0346-01610_12. MR2683245(2011m:34259) 
[16] M. Klaus and J. K. Shaw, On the eigenvalues of Zakharov-Shabat systems, SIAM J. Math. Anal. 34 (2003), no. 4, 759-773, DOI 10.1137/S0036141002403067. MR1969601 (2004c:34260)

[17] Jérôme Jp. Leon, The Dirac inverse spectral transform: kinks and boomerons, J. Math. Phys. 21 (1980), no. 10, 2572-2578, DOI 10.1063/1.524362. MR586340(82d:81022)

[18] C.J. Pethick and H. Smith, Bose-Einstein Condensation in Dilute Gases, Cambridge University Press, Cambridge (2002).

[19] A. Fratalocchi, C. Conti, G.Ruocco, S. Trillo Free-energy transition in a gas of noninteracting nonlinear wave particles, Phys. Rev. Lett. 101: 044101, (2008).

[20] C. van der Mee, Nonlinear Evolution Models of Integrable Type, 100 pages, SIMAI e-Lecture Notes 11, 2013.

[21] V. E. Zakharov and A. B. Shabat, Exact theory of two-dimensional self-focusing and onedimensional self-modulation of waves in nonlinear media (Russian, with English summary), Ž. Èksper. Teoret. Fiz. 61 (1971), no. 1, 118-134; English transl., Soviet Physics JETP 34 (1972), no. 1, 62-69. MR0406174 (53 \#9966)

[22] V.E. Zakharov and A.B. Shabat, Interaction between solitons in a stable medium, Sov. Phys. JETP 37, 823-828 (1973).

Università di Cagliari, Dip. Matematica e Informatica, viale Merello 92, Cagliari E-mail address: fdemontis@unica.it

Università di Cagliari, Dip. Matematica e Informatica, viale Merello 92, Cagliari E-mail address: cornelis@krein.unica.it

Università del Salento, Dip. Matematica e Fisica "E. De Giorgi”, via per Arnesano, LECCE AND SEZIONE INFN

E-mail address: federica.vitale@le.infn.it 